\title{
ON A CHARACTERISTIC PROPERTY OF LINEAR FUNCTIONS
}

\author{
E. F. BECKENBACH
}

1. Introduction. If the real function $y=g(x)$, defined and continuous in the closed and bounded interval $a \leqq x \leqq b$, or in the open interval $a<x<b$, is linear there,

$$
y=p x+q,
$$

then for all $x_{0}, h$, with $h>0$, such that $x_{0}-h$ and $x_{0}+h$ lie in the interval of definition, we have

$$
g\left(x_{0}\right)=\left[g\left(x_{0}-h\right)+g\left(x_{0}+h\right)\right] / 2 .
$$

Conversely, if the real function $y=g(x)$, defined and continuous in $a \leqq x \leqq b$ or in $a<x<b$, satisfies (1) for all $x_{0}, h$, with $h>0$, such that $x_{0}-h$ and $x_{0}+h$ lie in the interval of definition, then $\left[4\right.$, p. 189] ${ }^{1}$ $y=g(x)$ is a linear function of $x$.

If, however, in the converse it is given only that for each $x_{0}$, $a<x_{0}<b$, there exists a positive $h_{0}=h_{0}\left(x_{0}\right)$, such that $x_{0}-h_{0}$ and $x_{0}+h_{0}$ lie in the interval of definition, and for which we have

$$
g\left(x_{0}\right)=\left[g\left(x_{0}-h_{0}\right)+g\left(x_{0}+h_{0}\right)\right] / 2,
$$

then the implications are different in the case that $g(x)$ is defined and continuous in the closed and bounded interval and in the case that $g(x)$ is defined and continuous only in the open interval; for in the former case it still follows [3, p. 253] that $g(x)$ must be linear, while in the latter case $g(x)$ is not necessarily linear [3, pp. 253-255].

A proof of the above result, that if $g(x)$ is defined and continuous in the closed and bounded interval and satisfies (2) then $g(x)$ necessarily is linear, can be given, as we shall show, which applies equally well to characterize, in terms of equalities analogous to (2), classes of functions [1] differing, and even topologically distinct [2], from the class of linear functions.

2. Theorem. We shall establish the following result.

THEOREM. Let $\{f(x)\}$ be a class of functions defined and continuous in the closed and bounded interval $a \leqq x \leqq b$, and such that for all real $\left(x_{1}, y_{1}\right),\left(x_{2}, y_{2}\right)$ with $a \leqq x_{1}<x_{2} \leqq b$ there is a unique member 1945.

Presented to the Society, November 24, 1945; received by the editors April 24,

${ }^{1}$ Numbers in brackets refer to the references cited at the end of the paper. 


$$
f(x) \equiv f\left(x ; x_{1}, y_{1} ; x_{2}, y_{2}\right)
$$

of the family $\{f(x)\}$ satisfying

$$
f\left(x_{1}\right)=y_{1}, \quad f\left(x_{2}\right)=y_{2} .
$$

If the real function $g(x)$ is defined and continuous in the interval $a \leqq x \leqq b$ and if for each $x_{0}$ satisfying $a<x_{0}<b$ there is a positive $h_{0}=h_{0}\left(x_{0}\right)$, $a \leqq x_{0}-h_{0}<x_{0}+h_{0} \leqq b$, such that

$$
g\left(x_{0}\right)=f\left[x_{0} ; x_{0}-h_{0}, g\left(x_{0}-h_{0}\right) ; x_{0}+h_{0}, g\left(x_{0}+h_{0}\right)\right],
$$

then $g(x)$ coincides with a member of the family $\{f(x)\}$.

The above theorem contains as special case the result concerning linear functions mentioned in the last paragraph of $\$ 1$.

The converse of the theorem clearly holds, so that condition (3) is both necessary and sufficient in order that a function defined and continuous in the closed and bounded interval be a member of $\{f(x)\}$.

3. Lemmas. We shall use the following result [1].

LEMMA 1. For a given $x_{0}$ with $a \leqq x_{0} \leqq b$, let $f_{r}(x)$ and $f_{8}(x)$ be two members of the family $\{f(x)\}$, such that

$$
f_{r}\left(x_{0}\right)=f_{8}\left(x_{0}\right)
$$

and

$$
f_{r}(x) \not \equiv f_{s}(x),
$$$$
a \leqq x \leqq b ;
$$

then $f_{r}(x)<f_{s}(x)$ for all $x$ in $a \leqq x \leqq b$ on one side of $x_{0}$, while $f_{r}(x)>f_{s}(x)$ for all $x$ in $a \leqq x \leqq b$ on the other side of $x_{0}$.

Proof. By (4), (5) and the uniqueness property of the family $\{f(x)\}$, we have $f_{r}(x) \neq f_{s}(x)$ in $a \leqq x \leqq b$ except at $x_{0}$. Consequently, by the continuity of the members of $\{f(x)\}$, on either side of $x_{0}$ in $a \leqq x \leqq b$, one of $f_{r}(x)$ and $f_{s}(x)$ is greater than the other.

If $x_{0}=a$ or $x_{0}=b$, the theorem now follows.

If $a<x_{0}<b$, we shall obtain a contradiction from the assumption that it could be the same one of $f_{r}(x)$ and $f_{s}(x)$, say $f_{s}(x)$, which is greater on each side of $x_{0}$.

Let $x_{1}, x_{2}$ satisfy $a<x_{1}<x_{0}<x_{2}<b$, and consider the member $f_{t}(x)$ of $\{f(x)\}$, determined by

$$
f_{t}(x) \equiv f\left[x ; x_{1}, f_{8}\left(x_{1}\right) ; x_{2}, f_{r}\left(x_{2}\right)\right] .
$$

We have $f_{t}\left(x_{2}\right)<f_{s}\left(x_{2}\right)$, so that $f_{t}(x)<f_{s}(x), x_{1}<x \leqq b$; in particular,

$$
f_{t}\left(x_{0}\right)<f_{s}\left(x_{0}\right) \text {. }
$$


Similarly,

$$
f_{t}\left(x_{0}\right)>f_{r}\left(x_{0}\right)
$$

Now (6) and (7) contradict (4).

We shall use also the following lemma.

Lemma 2. For any positive $M$ there is a member $f^{\prime}(x)$ of $\{f(x)\}$ such that

$$
f^{\prime}(x)>M, \quad a \leqq x \leqq b .
$$

Proof. By the continuity of the members of $\{f(x)\}$, there is a positive $h$ such that if $I(a)$ and $I(b)$ denote the intervals $a \leqq x<a+h$ and $b-h<x \leqq b$ respectively, then we have

$$
f_{0}^{\prime}(x) \equiv f[x ; a, 2 M ; b, 2 M]>M
$$

for all $x$ in $I(a)$ and for all $x$ in $I(b)$. Similarly, for each $x^{\prime}$ in $a<x<b$ there is a positive $h=h\left(x^{\prime}\right)$ such that if $I\left(x^{\prime}\right)$ denotes the interval $x^{\prime}-h<x<x^{\prime}+h$, then we have

$$
f\left[x ; a, 2 M ; x^{\prime}, 2 M\right]>M
$$

for all $x$ in $I\left(x^{\prime}\right)$.

By the Heine-Borel theorem there is a finite set $a, b, x_{1}^{\prime}, \cdots, x_{n}^{\prime}$ of numbers in $a \leqq x \leqq b$ such that each number in $a \leqq x \leqq b$ is contained in at least one of $I(a), I(b), I\left(x_{1}^{\prime}\right), \cdots, I\left(x_{n}^{\prime}\right)$. Hence there is a finite set

$$
f_{0}^{\prime}(x), f_{1}^{\prime}(x), \cdots, f_{n}^{\prime}(x)
$$

of functions in the left-hand members of (9) and (10) such that for each $x$ in $a \leqq x \leqq b$ we have

$$
\max \left[f_{0}^{\prime}(x), f_{1}^{\prime}(x), \cdots, f_{n}^{\prime}(x)\right]>M .
$$

If now $f^{\prime}(x)$ is one of the functions (11), such that

$$
f^{\prime}(b)=\max \left[f_{0}^{\prime}(b), f_{1}^{\prime}(b), \cdots, f_{n}^{\prime}(b)\right],
$$

then since $f_{j}^{\prime}(a)=2 M, j=0,1, \cdots, m$, it follows from (12) and Lemma 1 that $f^{\prime}(x)$ satisfies (8).

4. A Dedekind section. Let $f_{0}(x)$ be the member of $\{f(x)\}$ coinciding with $g(x)$ at $x=a$ and at $x=b$; that is,

$$
f_{0}(x) \equiv f[x ; a, g(a) ; b, g(b)],
$$

and consider the members $f_{\alpha}(x)$ of $\{f(x)\}$ defined, for $-\infty<\alpha<+\infty$, by 


$$
f_{\alpha}(a)=g(a)+\alpha=f_{0}(a)+\alpha, f_{\alpha}(b)=g(b)+\alpha=f_{0}(b)+\alpha .
$$

Let a Dedekind section $(A, B)$ of the real numbers be defined as follows: $B$ contains all real numbers $\beta$ such that

$$
f_{\beta}(x)>g(x), \quad a \leqq x \leqq b,
$$

and $A$ contains all other real numbers $\alpha$.

To see that we actually have defined a Dedekind section of the real numbers, we note first that by definition all real numbers are included in $A$ and $B$ together. Further, $A$ is not vacuous; for $f_{0}(x)$ coincides with $g(x)$ at $x=a$, so that 0 is contained in $A$. And by Lemma 2, $B$ is not vacuous, since the function $g(x)$, continuous in the closed and bounded interval $a \leqq x \leqq b$, is bounded there. Finally, each $\alpha$ of $A$ is less than each $\beta$ of $B$; for if we should have $\beta_{0}<\alpha_{0}$, then by Lemma 1 we would have

$$
f_{\beta_{0}}(x)<f_{\alpha_{0}}(x), \quad a \leqq x \leqq b,
$$

and therefore, by the definition of $B$,

$$
g(x)<f_{\beta_{0}}(x)<f_{\alpha_{0}}(x), \quad a \leqq x \leqq b,
$$

and $\alpha_{0}$ would be a member of $B$.

Hence $(A, B)$ is a Dedekind section of the real numbers and determines a real number $\gamma$. Since 0 is contained in $A$, it follows that

$$
\gamma \geqq 0 \text {. }
$$

In $\$ 6$ we shall show that $\gamma=0$.

5. Properties of a function determined by the section. The function $f_{\gamma}(x)$ has the following properties:

$$
\begin{array}{ll}
f_{\gamma}(x) \geqq g(x), & a \leqq x \leqq b ; \\
f_{\gamma}\left(x_{0}\right)=g\left(x_{0}\right) &
\end{array}
$$

for some $x_{0}, a \leqq x_{0} \leqq b$.

If (15) did not hold, by (13) and (14) there would be an $x_{1}, a<x_{1}<b$, such that

$$
f_{\gamma}\left(x_{1}\right)<g\left(x_{1}\right)
$$

Consider the functions

$$
f^{(1)}(x) \equiv f\left[x ; a, f_{\gamma}(a) ; x_{1}, 2 f_{\gamma}\left(x_{1}\right) / 3+g\left(x_{1}\right) / 3\right]
$$

and

$$
f^{(2)}(x) \equiv f\left[x ; x_{1}, f_{\gamma}\left(x_{1}\right) / 3+2 g\left(x_{1}\right) / 3 ; b, f^{(1)}(b)\right]
$$


and let $\delta$ be defined by

$$
\delta=\min \left[f^{(2)}(a)-g(a), f^{(2)}(b)-g(b)\right] .
$$

By (17), (18), (19) and Lemma 1 , we have $\delta>\gamma$, so that $\delta$ is contained in the set $B$. But by (17), (19), (20) and Lemma 1, we have

$$
f_{\delta}\left(x_{1}\right)<g\left(x_{1}\right)
$$

whence, by the definition of $A, \delta$ is contained in the set $A$. But $\delta$ can not be contained both in $A$ and in $B$; this contradiction establishes (15).

If (16) did not hold, by (15) we would have

$$
f_{\gamma}(x)>g(x),
$$$$
a \leqq x \leqq b .
$$

Since the members of $\{f(x)\}$ are continuous, by (21) there is a positive $k$ such that if $J(a)$ and $J(b)$ denote the intervals $a \leqq x<a+k$ and $b-k<x \leqq b$ respectively, then

$$
f_{0}^{*}(x) \equiv f\left[x ; a, f_{\gamma}(a) ; b, g(b) / 2+f_{\gamma}(b) / 2\right]>g(x)
$$

for all $x$ in $J(a)$ and for all $x$ in $J(b)$. Similarly, for each $x^{*}$ in $a<x<b$ there is a positive $k=k\left(x^{*}\right)$ such that if $J\left(x^{*}\right)$ denotes the interval $x^{*}-k<x<x^{*}+k$, then

$$
f\left[x ; a, f_{\gamma}(a) ; x^{*}, g\left(x^{*}\right) / 2+f_{\gamma}\left(x^{*}\right) / 2\right]>g(x)
$$

for all $x$ in $J\left(x^{*}\right)$.

By the Heine-Borel theorem there is a finite set $a, b, x_{1}{ }^{*}, \cdots, x_{m}{ }^{*}$ of numbers in $a \leqq x \leqq b$ such that each number in $a \leqq x \leqq b$ is contained in at least one of $J(a), J(b), J\left(x_{1}^{*}\right), \cdots, J\left(x_{m}{ }^{*}\right)$. Hence there is a finite set

$$
f_{0}^{*}(x), f_{1}^{*}(x), \cdots, f_{m}^{*}(x)
$$

of functions in the left-hand members of (22) and (23) such that for each $x$ in $a \leqq x \leqq b$ we have

$$
\max \left[f_{0}^{*}(x), f_{1}^{*}(x), \cdots, f_{m}^{*}(x)\right]>g(x) .
$$

If now $f^{*}(x)$ is one of the functions (24) such that

$$
f^{*}(b)=\max \left[f_{0}^{*}(b), f_{1}^{*}(b), \cdots, f_{m}^{*}(b)\right],
$$

then since $f_{j}^{*}(a)=f_{\gamma}(a), j=0,1, \cdots, m$, it follows from (25) and Lemma 1 that $f^{*}(x)$ satisfies

$$
f^{*}(x)>g(x), \quad a \leqq x \leqq b .
$$

By (21), (22), (23) and Lemma 1, we have 


$$
f_{j}^{*}(x)<f_{\gamma}(x), \quad j=0,1, \cdots, m ; a<x \leqq b ;
$$

in particular,

$$
f^{*}(x)<f_{\gamma}(x), \quad a<x \leqq b,
$$

with

$$
f^{*}(a)=f_{\gamma}(a)
$$

Starting with (26) in place of (21), and interchanging the rôles of $a$ and $b$, we can determine analogously the existence of a member $f^{\prime \prime}(x)$ of $\{f(x)\}$, such that

$$
f^{\prime \prime}(x)>g(x), \quad a \leqq x \leqq b,
$$

and

$$
f^{\prime \prime}(x)<f^{*}(x), \quad a \leqq x<b,
$$

with

$$
f^{\prime \prime}(b)=f^{*}(b) .
$$

From (27), (28), (30) and (31) we obtain

$$
f^{\prime \prime}(x)<f_{\gamma}(x),
$$$$
a \leqq x \leqq b .
$$

Let $\epsilon$ be defined by

$$
\epsilon=\max \left[f^{\prime \prime}(a)-g(a), f^{\prime \prime}(b)-g(b)\right] .
$$

By (29) and (32) we have

$$
0<\epsilon<\gamma .
$$

Hence from (29), (33) and Lemma 1 we obtain

$$
f_{\epsilon}(x)>g(x), \quad a \leqq x \leqq b,
$$

so that $\epsilon$ is contained in $B$. But by (34) and the definition of $\gamma, \epsilon$ is contained in $A$; this contradiction establishes (16).

We note that (15) and (16) might also have been established by proving first that

$$
\lim _{\zeta \rightarrow \gamma} f_{\zeta}(x)=f_{\gamma}(x), \quad a \leqq x \leqq b .
$$

6. The value of $\gamma$. By (14) we have either

$$
\gamma=0
$$

or

$$
\gamma>0 .
$$


If (36) holds, then by the definition of $f_{\gamma}(x)$ we have

$$
f_{\gamma}(a)>g(a), \quad f_{\gamma}(b)>g(b) .
$$

By the continuity of $f_{\gamma}(x)$ and of $g(x)$, there is a greatest number $x_{0}$ in $a \leqq x \leqq b$ satisfying (16); and by (37), this greatest $x_{0}$ satisfies $a<x_{0}<b$. Then

$$
f_{\gamma}(x)>g(x), \quad x_{0}<x \leqq b .
$$

By hypothesis there is a positive number $h_{0}=h_{0}\left(x_{0}\right), a \leqq x_{0}-h_{0}$ $<x_{0}+h_{0} \leqq b$, for which (3) is satisfied.

From (15) we obtain

$$
f_{\gamma}\left(x_{0}-h_{0}\right) \geqq g\left(x_{0}-h_{0}\right),
$$

and from (38) we get

$$
f_{\gamma}\left(x_{0}+h_{0}\right)>g\left(x_{0}+h_{0}\right) .
$$

Hence by Lemma 1 we have

$$
f_{\gamma}(x)>f\left[x ; x_{0}-h_{0}, g\left(x_{0}-h_{0}\right) ; x_{0}+h_{0}, g\left(x_{0}+h_{0}\right)\right]
$$

for $x_{0}-h<x \leqq x_{0}+h$; in particular,

$$
f_{\gamma}\left(x_{0}\right)>f\left[x_{0} ; x_{0}-h_{0}, g\left(x_{0}-h_{0}\right) ; x_{0}+h_{0}, g\left(x_{0}+h_{0}\right)\right] .
$$

Now (39) contradicts (3) and (16), so that (36) does not hold, and therefore the value of $\gamma$ is given by (35).

7. Proof of the theorem. We shall establish the theorem by showing that

$$
g(x) \equiv f_{0}(x), \quad a \leqq x \leqq b .
$$

Suppose that (40) does not hold; that is, that we have

$$
g(x) \not \equiv f_{0}(x),
$$$$
a \leqq x \leqq b .
$$

We shall obtain a contradiction.

By (13) and (41) there is an $\bar{x}, a<\bar{x}<b$, such that

$$
g(\bar{x}) \neq f_{0}(\bar{x}) \text {; }
$$

and we can suppose that

$$
g(\bar{x})>f_{0}(\bar{x}),
$$

since otherwise we could consider the function $-g(x)$ and the family $\{-f(x)\}$.

From (15) and (35) we obtain 


$$
f_{0}(x) \geqq g(x),
$$$$
a \leqq x \leqq b .
$$

Since (43) contradicts (42), the assumption that (40) does not hold has led to a contradiction.

\section{REFERENCES}

1. E. F. Beckenbach, Generalized convex functions, Bull. Amer. Math. Soc. vol. 43 (1937) pp. 363-371.

2. E. F. Beckenbach and R. H. Bing, On generalized convex functions, Trans. Amer. Math. Soc. vol. 58 (1945) pp. 220-230.

3. R. Courant und D. Hilbert, Methoden der mathematischen Physik, Berlin, 1937.

4. J. L. W. V. Jensen, Sur les fonctions convexes et les inégalitês entre les valeurs moyennes, Acta Math. vol. 30 (1906) pp. 175-193.

The University of Texas

\section{NOTE ON A CERTAIN CONTINUED FRACTION}

\section{H. S. WALL}

The continued fraction

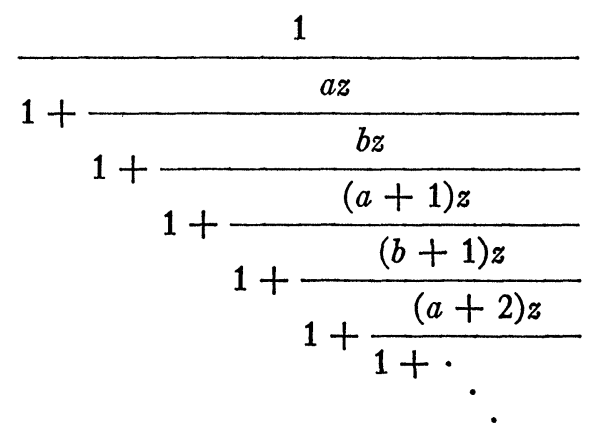

is a limiting case of the continued fraction of Gauss, and is the formal expansion of the quotient $\Omega(a, b ; z) / \Omega(a, b-1 ; z)$, where

$$
\Omega(a, b ; z)=1-a b \frac{z}{1 !}+a(a+1) b(b+1) \frac{z^{2}}{2 !}+\cdots .
$$

If $a$ and $b$ are real and positive, then it follows from the work of Stieltjes that (1) converges in the domain $Z$ exterior to the negative 